Prepared in cooperation with the Kansas Department of Agriculture, Division of Water Resources

\title{
Irrigation Water Use in Kansas, 2013
}

Data Series 981 



\section{Irrigation Water Use in Kansas, 2013}

By Jennifer L. Lanning-Rush

Prepared in cooperation with the Kansas Department of Agriculture, Division of Water Resources

Data Series 981

U.S. Department of the Interior

U.S. Geological Survey 


\title{
U.S. Department of the Interior SALLY JEWELL, Secretary
}

\section{U.S. Geological Survey Suzette M. Kimball, Director}

\author{
U.S. Geological Survey, Reston, Virginia: 2016
}

For more information on the USGS - the Federal source for science about the Earth, its natural and living resources, natural hazards, and the environment—visit http://www.usgs.gov or call 1-888-ASK-USGS.

For an overview of USGS information products, including maps, imagery, and publications, visit http://www.usgs.gov/pubprod/.

Any use of trade, firm, or product names is for descriptive purposes only and does not imply endorsement by the U.S. Government.

Although this information product, for the most part, is in the public domain, it also may contain copyrighted materials as noted in the text. Permission to reproduce copyrighted items must be secured from the copyright owner.

Suggested citation:

Lanning-Rush, J.L., 2016, Irrigation water use in Kansas, 2013: U.S. Geological Survey Data Series 981, 12 p., http://dx.doi.org/10.3133/ds981.

ISSN 2327-638X (online) 


\section{Contents}

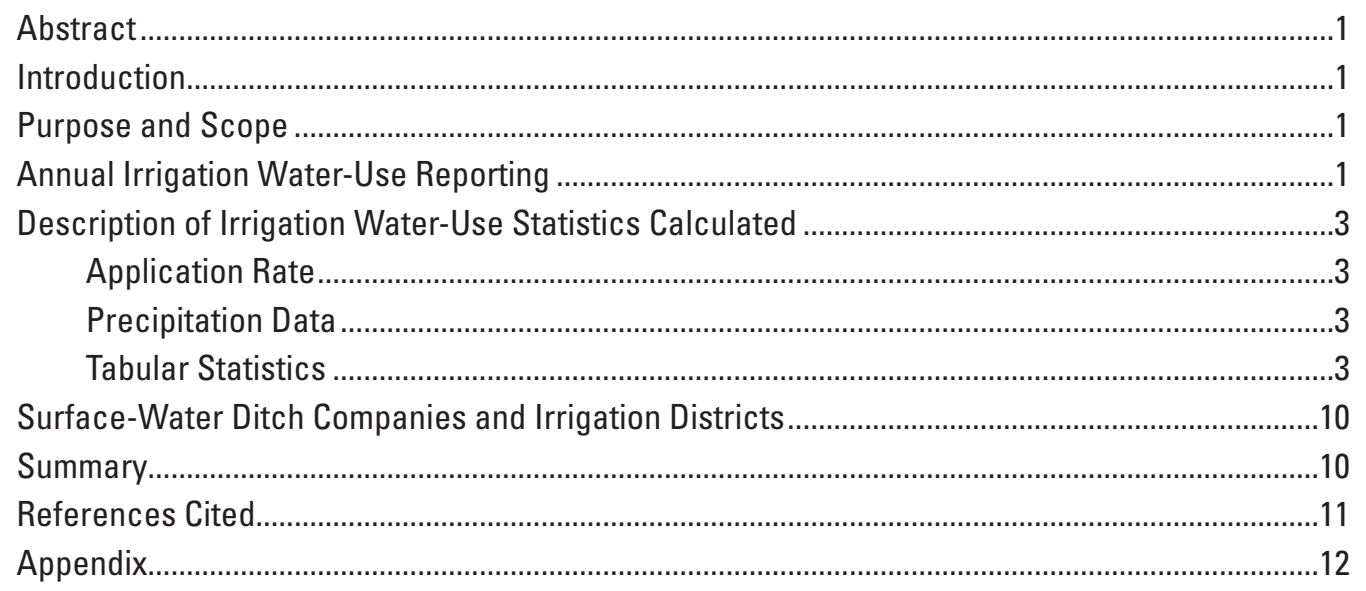

\section{Figures}

1. Map showing Kansas irrigation water-use analysis regions...........................................

2. Map showing Kansas regional planning areas used in the Kansas Water Plan...............2

\section{Tables}

1. Water-use and precipitation statistics by Kansas irrigation water-use analysis

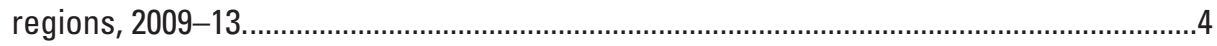

2. Water-use and precipitation statistics by Kansas regional planning areas, 2013.............5

3. Water-use and precipitation statistics by county, 2009-13 …...........................................

4. Comparison of water use and acres irrigated by meter status and Kansas

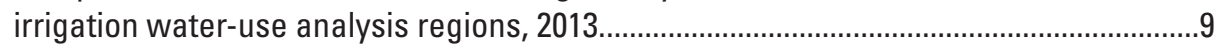

5. Comparison of water use and acres irrigated by meter status and regional

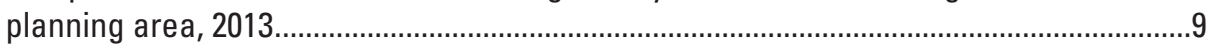

6. Water use, acres irrigated, and average application rate by crop and Kansas

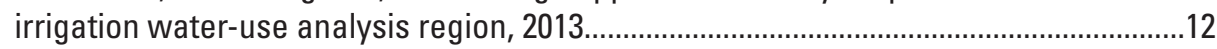

7. Water use, acres irrigated, and average application rate by crop and regional planning area, 2013....................................................................................................12

8. Water use, acres irrigated, and average application rate by crop and county, 2013.....12

9. Water use, acres irrigated, and average application rate by type of irrigation system by Kansas irrigation water-use analysis regions, 2013 ….................................12

10. Water use, acres irrigated, and average application rate by type of irrigation system by regional planning area, 2013.......................................................................12

11. Water use, acres irrigated, and average application rate by type of irrigation

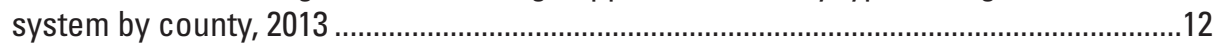

12. Water use and acres irrigated by ditch companies and irrigation districts in Kansas, 2013 


\section{Conversion Factors}

U.S. customary units to International System of Units

\begin{tabular}{lcl}
\hline \multicolumn{1}{c}{ Multiply } & By & \multicolumn{1}{c}{ To obtain } \\
\hline inch (in.) & Length & \\
inch (in.) & 2.54 & centimeter $(\mathrm{cm})$ \\
\hline & 25.4 & millimeter $(\mathrm{mm})$ \\
\hline acre & Area & \\
acre & 4,047 & square meter $\left(\mathrm{m}^{2}\right)$ \\
square mile $\left(\mathrm{mi}^{2}\right)$ & 0.004047 & square kilometer $\left(\mathrm{km}^{2}\right)$ \\
square mile $\left(\mathrm{mi}^{2}\right)$ & 259.0 & hectare $($ ha) \\
\hline & 2.590 & square kilometer $\left(\mathrm{km}^{2}\right)$ \\
\hline gallon (gal) & Volume & \\
gallon (gal) & 3.785 & liter $(\mathrm{L})$ \\
gallon (gal) & 0.003785 & cubic meter $\left(\mathrm{m}^{3}\right)$ \\
million gallons (Mgal) & 3.785 & cubic decimeter $\left(\mathrm{dm}^{3}\right)$ \\
acre-foot (acre-ft) & 3,785 & cubic meter $\left(\mathrm{m}^{3}\right)$ \\
\hline
\end{tabular}




\title{
Irrigation Water Use in Kansas, 2013
}

\author{
By Jennifer L. Lanning-Rush
}

\begin{abstract}
This report, prepared by the U.S. Geological Survey in cooperation with the Kansas Department of Agriculture, Division of Water Resources, presents derivative statistics of 2013 irrigation water use in Kansas. The published regional and county-level statistics from the previous 4 years (2009-12) are shown with the 2013 statistics and are used to calculate a 5-year average. An overall Kansas average and regional averages also are calculated and presented. Total reported irrigation water use in 2013 was 3.3 million acre-feet of water applied to 3.0 million irrigated acres.
\end{abstract}

\section{Introduction}

Agriculture is the largest land use in Kansas, accounting for approximately 88 percent of all Kansas land (National Agricultural Statistics Service, 2014). Irrigated agricultural land is by far the largest use of water in Kansas, typically representing about 3 million acres in the State (Kenny and Juracek, 2013). Kansas supports a variety of crops and consistently ranks in the top 10 nationally in wheat, grain sorghum, grain corn production, total acres irrigated, and total acres in cropland (Kansas Department of Agriculture, 2015). Irrigation is most prevalent in western Kansas where average annual rainfall is less than 20 inches and sufficient groundwater can be pumped to compensate for the lack of precipitation (High Plains Regional Climate Center, 2015). Groundwater-based irrigation water use is less in eastern Kansas, where precipitation is greater.

Kansas has two State agencies with responsibilities related to water-quantity planning: the Kansas Department of Agriculture, Division of Water Resources (DWR) and the Kansas Water Office. The DWR administers the Kansas Water Appropriation Act (Kansas Statutes Annotated § 82a-701 to 82a-726), which governs how water is allocated and used within the State through water rights and collects annual information on amounts of water used. The Kansas Water Office issues the State water plan, which is used to manage, conserve, and develop the water resources of the State. Data on irrigation water use have been published annually since the late 1980s and are a key component for the evaluation of conservation planning (Kansas Department of Agriculture, Division of Water Resources, 1989-2012).

\section{Purpose and Scope}

This report, prepared by the U.S. Geological Survey in cooperation with DWR with partial support from the State Water Plan Fund, presents reported water use, acres irrigated, and derivative statistics of 2013 irrigation water use in Kansas. Average application rates are calculated as a function of total irrigation withdrawals and total irrigated acres. Tabular statistics in this report are aggregated geographically by the Kansas irrigation water-use analysis regions (fig. 1), Kansas regional planning areas (fig. 2), and all 105 counties in the State (fig. 2).

\section{Annual Irrigation Water-Use Reporting}

A provision of the DWR's water appropriation program requires all irrigation water right owners to submit an annual water-use report on a paper form (an example is provided in the appendix). Data submitted on the water-use reports are stored in the DWR's Water Rights Information System (WRIS) (Kenny, 2014). The WRIS is the primary record-keeping and reporting mechanism for water rights and water-use information. Information is reported by point of diversion (the point from which water was obtained) and includes the quantity of water diverted each calendar year, type of crops irrigated, number of acres irrigated, and type of irrigation system (Kansas Department of Agriculture, Division of Water Resources, 2015). Each acre is counted only once regardless of the number of times it was irrigated or harvested. DWR staff send follow-up letters and make phone calls to obtain missing data and to confirm or correct anomalous data. 


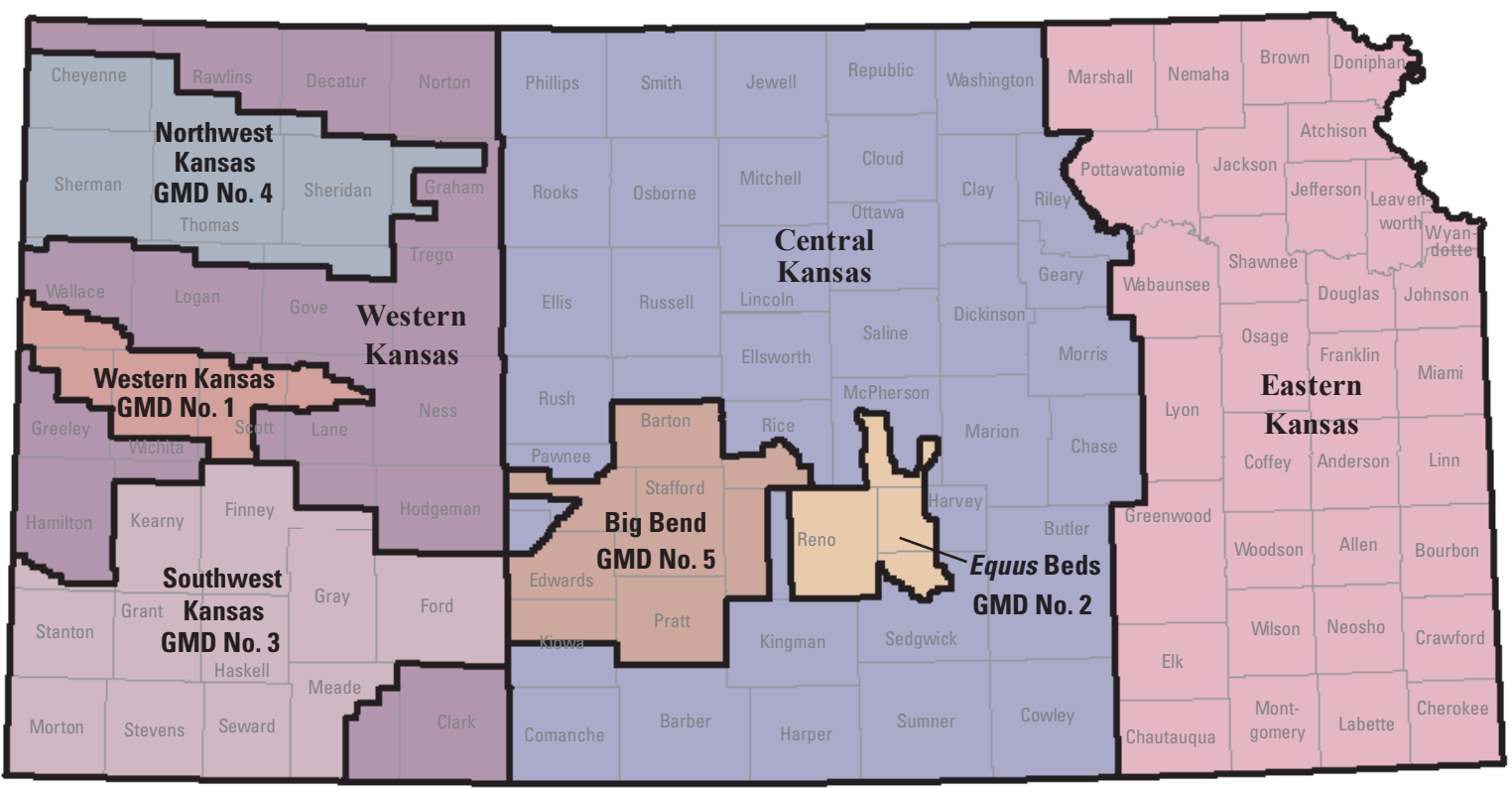

Figure 1. Kansas irrigation water-use analysis regions [GMD, groundwater management district].

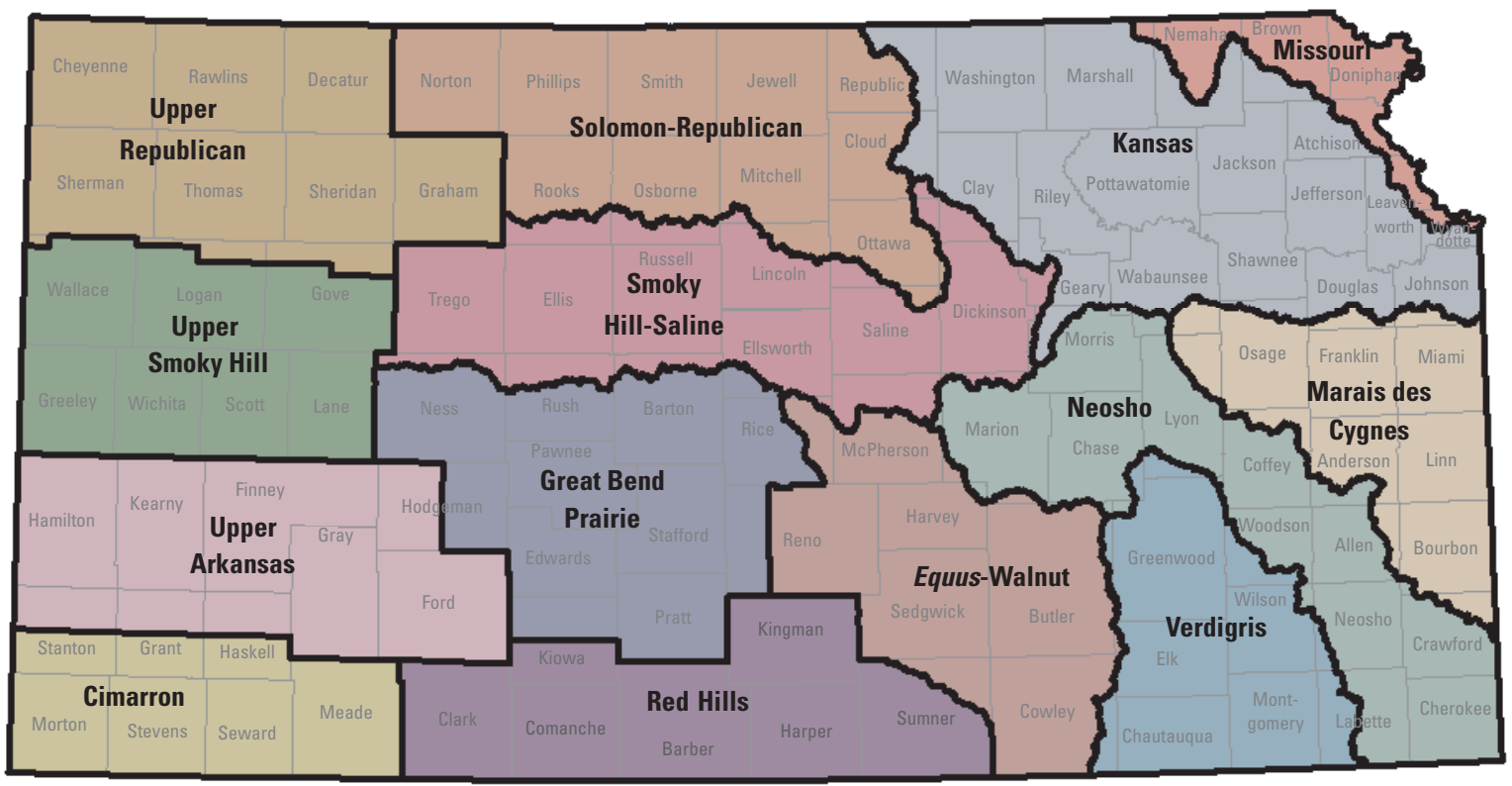

Figure 2. Kansas regional planning areas used in the Kansas Water Plan. 


\section{Description of Irrigation Water-Use Statistics Calculated}

\section{Application Rate}

Water used for irrigation is often described in terms of application rate, which is the amount of water used per acre of land irrigated. Application rate is often expressed as acrefeet per acre. Irrigation application rates can vary from year to year and are affected by precipitation, soil permeability, temperature, wind, crop type, length of irrigation season, and irrigation system type (Perry, 2006). Application rate is utilized in this report as a method of comparing irrigation water use intensity among regions and counties. Published statistics from the previous 4 years (2009-12) (Kansas Department of Agriculture, Division of Water Resources, 1989-2012) are also shown with the 2013 statistics and are used to calculate a 5 -year average for Kansas irrigation by regions and counties. Because 2013 is the first year that statistics have been computed for the regional planning areas, there are no statistics published from previous years with which to calculate a 5-year average. An overall Kansas average and regional averages also are calculated and presented. Although most of the irrigated lands in Kansas are in crops, some counties may have additional irrigation water use for golf courses, nurseries, and recreational areas.

\section{Precipitation Data}

Reported irrigation water withdrawals vary substantially from year to year as affected primarily by climatic fluctuations (Kenny and Juracek, 2013). Historically, Kansas has experienced large year-to-year variations in precipitation. At the beginning of 2013, the entire State of Kansas was categorized to be in severe drought, and over one-third of the State was classified in exceptional drought conditions (U.S. Drought Monitor, 2014). The persistence of the drought resulted in record-low 7-day average discharge measurements at five U.S. Geological Survey streamflow-gaging stations with more than 30 years of record (Peters and Rasmussen, 2014). By the end of 2013, there were no areas of extreme drought left in the State and only 33 percent were classified to be in any level of drought condition.

In this report, area-weighted statistical summarizations were performed on gridded precipitation data for 2013 annual total precipitation and the current 30-year climatic normal (based on 1981-2010) for the irrigation water-use analysis regions and regional planning areas in this report. Gridded precipitation data came from Oregon State University's Parameter-elevation Regressions on Independent Slopes Model (PRISM) (PRISM Climate Group, 2015). PRISM is an analytical model that generates gridded estimates of annual precipitation from point data at National Weather Service climatological stations and a digital elevation model (Di Luzio and others, 2008). The 2013 annual total precipitation and the current 30-year climatic normal (based on 1981-2010) for the 105 Kansas counties are from the Kansas statistical abstract 2013 (Institute for Policy and Social Research, 2014).

\section{Tabular Statistics}

Irrigation water-use statistics in this report are summarized by Kansas irrigation water-use analysis regions, Kansas regional planning areas, and the 105 counties within Kansas. Regional application rate averages for the years 2009, 2010, 2011, 2012, and 2013, as well as the 5-year average, are provided in table 1 by water-use analysis region. The 2013 average annual precipitation and the 30 -year normal precipitation, as well as the number of reporting and active points of diversion by water-use analysis region, are also shown on table 1 . The application rate for the year 2013 is provided in table 2 for the 14 regional planning areas along with the acres irrigated, water used, 2013 average annual precipitation, 30-year average annual precipitation, and the number of reporting and active points of diversion. The information in table 3 is identical to table 1 but aggregated to the county level.

Metered and nonmetered statistics are provided in table 4 by water-use analysis region (fig. 1). The instructions on the 2013 irrigation water-use report (see appendix for blank report) request that a beginning meter reading, an ending meter reading, a metered quantity, and the unit of measure be provided for water use if a water meter was installed. If a water meter had not been installed or the water meter did not work properly, then the irrigator is instructed to provide the number of hours pumped and the pumping rate for each point of diversion. In this report, it was assumed that all water is metered except where hours and pump rates are provided and meter trouble was not indicated on the report. The percent metered is computed and provided along with a count of the points of diversion reporting metered and nonmetered water use in 2013. The same statistics, aggregated by regional planning areas (fig. 2), are shown in table 5.

Application rates are further grouped by crop type. The instructions on the 2013 irrigation water-use report (see appendix for blank report) request that crops that were irrigated in 2013 be listed using the crop codes for each point of diversion. The crop codes are integer values for the following crops: alfalfa, corn, grain sorghum (milo), soybeans, wheat, oats, barley, rye, dry beans, sunflowers, golf course/ sports fields, truck farm, orchard, nursery, other, more than one type of crop, double crop, cotton, and grapes. The amount of water used, irrigated acres, and application rates for 2013 are provided in table 6 for alfalfa; corn; grain sorghum (milo); soybeans; wheat; combination of alfalfa, corn, grain sorghum, soybeans, or wheat; more than one type of crop specified; other; and those crops not specified on 2013 water-use report for the water-use analysis regions (fig. 1). Because it is common to irrigate multiple crop types with single points of diversion during a 12-month period, many irrigators list more than one of the crop-code values on their water-use report. For tabular statistics purposes, when an irrigator specified combinations of alfalfa, corn, grain sorghum (milo), or soybeans on a point of diversion, they were combined under the column 


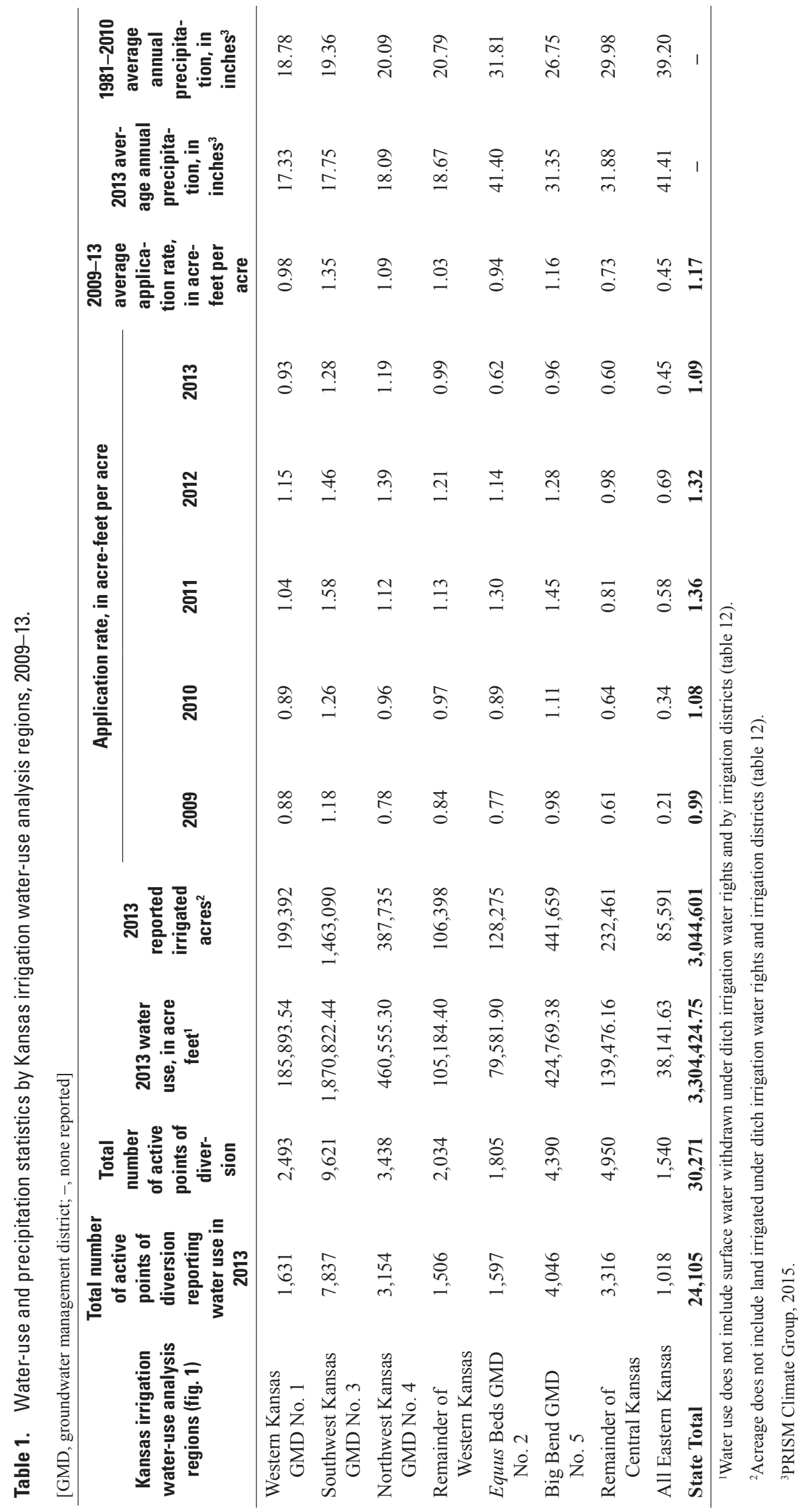


Table 2. Water-use and precipitation statistics by Kansas regional planning areas, 2013.

$[-$, none reported $]$

\begin{tabular}{|c|c|c|c|c|c|c|c|}
\hline $\begin{array}{l}\text { Regional planning area } \\
\text { (fig. 2) }\end{array}$ & $\begin{array}{l}\text { Total number of } \\
\text { active points of diver- } \\
\text { sion reporting water } \\
\text { use for the year } 2013\end{array}$ & $\begin{array}{l}\text { Total num- } \\
\text { ber of active } \\
\text { points of } \\
\text { diversion }\end{array}$ & $\begin{array}{l}2013 \text { water } \\
\text { use, in acre } \\
\text { feet }^{1}\end{array}$ & $\begin{array}{l}2013 \\
\text { reported } \\
\text { irrigated } \\
\text { acres }^{2}\end{array}$ & $\begin{array}{l}2013 \text { ap- } \\
\text { plication } \\
\text { rate, in } \\
\text { acre-feet } \\
\text { per acre }\end{array}$ & $\begin{array}{l}2013 \text { average } \\
\text { annual pre- } \\
\text { cipitation, in } \\
\text { inches }^{3}\end{array}$ & $\begin{array}{l}\text { 1981-2010 av- } \\
\text { erage annual } \\
\text { precipitation, } \\
\text { in inches }\end{array}$ \\
\hline Cimarron & 2,879 & 3,347 & $884,302.61$ & 668,044 & 1.32 & 16.67 & 18.90 \\
\hline Equus-Walnut & 2,148 & 2,487 & $92,464.76$ & 153,222 & 0.60 & 41.85 & 34.36 \\
\hline Great Bend Prairie & 4,640 & 5,198 & $459,144.04$ & 481,477 & 0.95 & 29.05 & 25.56 \\
\hline Kansas & 1,478 & 1,979 & $64,880.46$ & 133,251 & 0.49 & 34.10 & 34.86 \\
\hline Marais des Cygnes & 54 & 156 & $2,756.15$ & 5,981 & 0.46 & 43.74 & 40.69 \\
\hline Missouri & 102 & 120 & $3,834.34$ & 8,377 & 0.46 & 35.44 & 35.45 \\
\hline Neosho & 106 & 226 & $3,033.63$ & 8,855 & 0.34 & 43.90 & 39.59 \\
\hline Red Hills & 429 & 577 & $32,026.70$ & 38,961 & 0.82 & 30.68 & 28.87 \\
\hline Smoky Hill-Saline & 490 & 880 & $13,920.45$ & 26,129 & 0.53 & 29.17 & 27.20 \\
\hline Solomon-Republican & 1,221 & 1,881 & $50,627.48$ & 74,151 & 0.68 & 24.22 & 26.13 \\
\hline Upper Arkansas & 5,174 & 6,567 & $1,014,351.63$ & 819,594 & 1.24 & 17.59 & 19.68 \\
\hline Upper Republican & 3,528 & 3,901 & $483,556.42$ & 408,959 & 1.18 & 17.96 & 20.49 \\
\hline Upper Smoky Hill & 1,820 & 2,838 & $198,586.22$ & 214,329 & 0.93 & 18.15 & 19.06 \\
\hline Verdigris & 36 & 114 & 939.86 & 3,271 & 0.29 & 47.31 & 40.60 \\
\hline State Total & 24,105 & 30,271 & $3,304,424.75$ & $3,044,601$ & 1.09 & - & - \\
\hline
\end{tabular}

${ }^{1}$ Water use does not include surface water withdrawn under ditch irrigation water rights and by irrigation districts (table 12).

${ }^{2}$ Acreage does not include land irrigated under ditch irrigation water rights and irrigation districts (table 12).

${ }^{3}$ PRISM Climate Group, 2015. 
Table 3. Water-use and precipitation statistics by county, 2009-13.

$[-$, none reported $]$

\begin{tabular}{|c|c|c|c|c|c|c|c|c|c|c|c|c|}
\hline \multirow[b]{2}{*}{ County } & \multirow{2}{*}{$\begin{array}{c}\text { Total } \\
\text { number } \\
\text { of active } \\
\text { points of } \\
\text { diversion } \\
\text { reporting } \\
\text { water } \\
\text { use for } \\
\text { the year } \\
2013\end{array}$} & \multirow{2}{*}{$\begin{array}{l}\text { Total } \\
\text { num- } \\
\text { ber of } \\
\text { active } \\
\text { points } \\
\text { of } \\
\text { diver- } \\
\text { sion }\end{array}$} & \multirow[b]{2}{*}{$\begin{array}{c}2013 \text { water } \\
\text { use, in acre } \\
\text { feet }^{1}\end{array}$} & \multirow[b]{2}{*}{$\begin{array}{c}2013 \\
\text { reported } \\
\text { irrigated } \\
\text { acres }^{2}\end{array}$} & \multicolumn{5}{|c|}{ Application rate, in acre-feet per acre } & \multirow{2}{*}{$\begin{array}{c}2009-13 \\
\text { average } \\
\text { appli- } \\
\text { cation } \\
\text { rate, in } \\
\text { acre- } \\
\text { feet per } \\
\text { acre }\end{array}$} & \multirow{2}{*}{$\begin{array}{c}2013 \\
\text { average } \\
\text { annual } \\
\text { pre- } \\
\text { cipita- } \\
\text { tion, in } \\
\text { inches }^{3}\end{array}$} & \multirow{2}{*}{$\begin{array}{c}1981- \\
2010 \\
\text { average } \\
\text { annual } \\
\text { pre- } \\
\text { cipita- } \\
\text { tion, in } \\
\text { inches }^{3}\end{array}$} \\
\hline & & & & & 2009 & 2010 & 2011 & 2012 & 2013 & & & \\
\hline Allen & 4 & 11 & 30.02 & 240 & 0.42 & 0.36 & 0.61 & 0.49 & 0.13 & 0.40 & 43.73 & 43.38 \\
\hline Anderson & 12 & 23 & 765.77 & 1,963 & 0.33 & 0.32 & 0.59 & 0.45 & 0.39 & 0.42 & 34.06 & 40.00 \\
\hline Atchison & 15 & 18 & 270.36 & 766 & 0.29 & 0.27 & 0.26 & 0.70 & 0.35 & 0.37 & 33.39 & 36.97 \\
\hline Barber & 49 & 80 & $3,534.19$ & 4,186 & 0.95 & 1.00 & 1.60 & 1.27 & 0.84 & 1.13 & 25.01 & 28.51 \\
\hline Butler & 25 & 38 & 669.94 & 1,556 & 0.62 & 0.58 & 0.55 & 0.79 & 0.43 & 0.59 & 40.45 & 34.08 \\
\hline Chase & 1 & 15 & 1.53 & 11 & 0.03 & 0.03 & 0.00 & 0.09 & 0.14 & 0.06 & 38.85 & 34.91 \\
\hline Chautauqua & 2 & 12 & 0.86 & 120 & 0.00 & 0.00 & 0.24 & 0.55 & 0.01 & 0.16 & 44.60 & 38.42 \\
\hline Cherokee & 9 & 21 & 280.89 & 1,112 & 0.30 & 0.37 & 0.53 & 0.51 & 0.25 & 0.39 & 44.84 & 44.47 \\
\hline Cheyenne & 474 & 554 & $68,575.27$ & 47,439 & 0.90 & 1.00 & 1.18 & 1.54 & 1.45 & 1.21 & 14.18 & 18.35 \\
\hline Clark & 38 & 49 & $3,910.72$ & 4,604 & 0.76 & 0.94 & 1.26 & 1.21 & 0.85 & 1.00 & 21.02 & 23.18 \\
\hline Clay & 245 & 298 & $13,961.13$ & 24,929 & 0.45 & 0.35 & 0.42 & 0.85 & 0.56 & 0.53 & 28.91 & 30.29 \\
\hline Cloud & 283 & 332 & $11,888.98$ & 23,359 & 0.56 & 0.43 & 0.48 & 0.87 & 0.51 & 0.57 & 26.43 & 30.03 \\
\hline Coffey & 11 & 29 & 224.07 & 559 & 0.27 & 0.29 & 0.52 & 0.44 & 0.40 & 0.38 & 31.22 & 37.91 \\
\hline Doniphan & 19 & 21 & $1,229.53$ & 2,812 & 0.15 & 0.06 & 0.06 & 0.52 & 0.44 & 0.25 & 37.55 & 37.43 \\
\hline Douglas & 40 & 54 & $1,539.55$ & 2,957 & 0.18 & 0.40 & 0.55 & 0.74 & 0.52 & 0.48 & 30.86 & 39.33 \\
\hline Edwards & 855 & 929 & $97,623.39$ & 93,781 & 1.03 & 1.11 & 1.43 & 1.28 & 1.04 & 1.18 & 23.64 & 26.75 \\
\hline Elk & 1 & 1 & 2.45 & 4 & 0.00 & 0.00 & 0.00 & 0.01 & 0.61 & 0.12 & 43.63 & 38.91 \\
\hline Ellis & 54 & 124 & $1,307.95$ & 1,750 & 0.81 & 0.89 & 1.02 & 1.14 & 0.75 & 0.92 & 19.73 & 23.06 \\
\hline Ellsworth & 11 & 27 & 175.71 & 499 & 0.22 & 0.49 & 0.54 & 0.63 & 0.35 & 0.45 & 37.07 & 27.99 \\
\hline Finney & 1,461 & 1,733 & $298,826.84$ & 213,246 & 1.17 & 1.35 & 1.62 & 1.59 & 1.40 & 1.43 & 17.23 & 19.90 \\
\hline Ford & 666 & 847 & $88,907.55$ & 84,816 & 1.08 & 1.06 & 1.43 & 1.27 & 1.05 & 1.18 & 23.11 & 22.93 \\
\hline Franklin & 15 & 48 & $1,070.98$ & 2,465 & 0.67 & 0.86 & 0.96 & 0.72 & 0.43 & 0.73 & 29.15 & 38.28 \\
\hline Geary & 46 & 74 & $2,355.23$ & 4,787 & 0.47 & 0.50 & 0.64 & 0.89 & 0.49 & 0.60 & 31.49 & 32.54 \\
\hline Gove & 245 & 348 & $20,119.26$ & 20,597 & 0.73 & 0.90 & 1.05 & 1.23 & 0.98 & 0.98 & 17.24 & 22.92 \\
\hline Graham & 157 & 214 & $15,878.77$ & 14,287 & 0.68 & 1.03 & 0.85 & 1.29 & 1.11 & 0.99 & 14.97 & 21.90 \\
\hline Grant & 516 & 701 & $133,110.04$ & 104,733 & 1.19 & 1.32 & 1.55 & 1.46 & 1.27 & 1.36 & 15.41 & 17.39 \\
\hline Gray & 1,134 & 1,437 & $194,516.16$ & 165,065 & 1.12 & 1.21 & 1.52 & 1.35 & 1.18 & 1.28 & 14.99 & 23.05 \\
\hline Greeley & 163 & 266 & $20,609.66$ & 24,002 & 0.97 & 0.88 & 1.04 & 1.23 & 0.86 & 1.00 & 15.81 & 17.44 \\
\hline Greenwood & 5 & 13 & 152.58 & 142 & 0.50 & 0.43 & 0.45 & 0.69 & 1.07 & 0.63 & 37.60 & 36.71 \\
\hline
\end{tabular}


Table 3. Water-use and precipitation statistics by county, 2009-13._-Continued

$[-$, none reported $]$

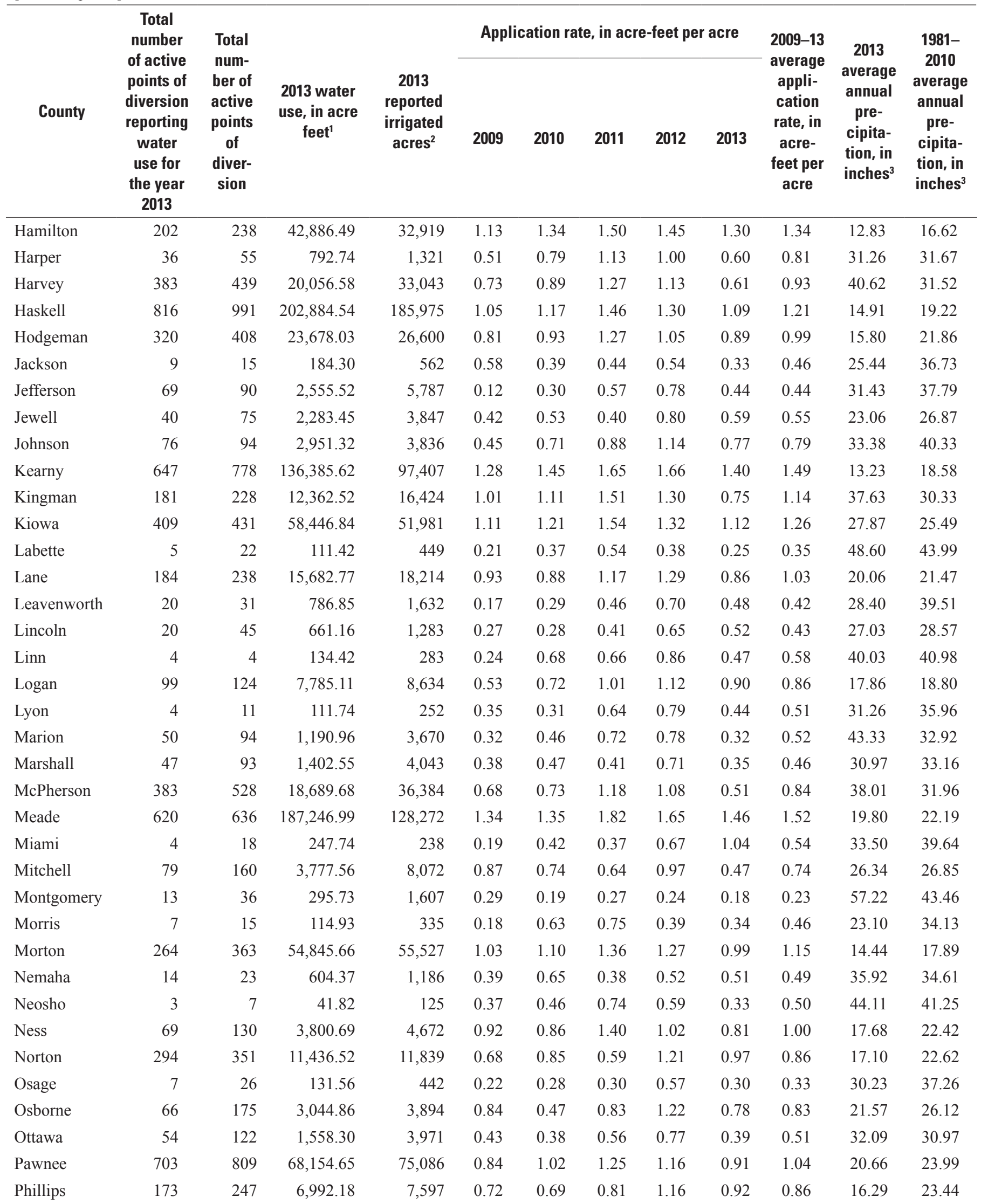


Table 3. Water-use and precipitation statistics by county, 2009-13._-Continued

$[-$, none reported $]$

\begin{tabular}{|c|c|c|c|c|c|c|c|c|c|c|c|c|}
\hline \multirow[b]{2}{*}{ County } & \multirow{2}{*}{$\begin{array}{c}\text { Total } \\
\text { number } \\
\text { of active } \\
\text { points of } \\
\text { diversion } \\
\text { reporting } \\
\text { water } \\
\text { use for } \\
\text { the year } \\
2013\end{array}$} & \multirow{2}{*}{$\begin{array}{l}\text { Total } \\
\text { num- } \\
\text { ber of } \\
\text { active } \\
\text { points } \\
\text { of } \\
\text { diver- } \\
\text { sion }\end{array}$} & \multirow[b]{2}{*}{$\begin{array}{l}2013 \text { water } \\
\text { use, in acre } \\
\text { feet }^{1}\end{array}$} & \multirow[b]{2}{*}{$\begin{array}{c}2013 \\
\text { reported } \\
\text { irrigated } \\
\text { acres }^{2}\end{array}$} & \multicolumn{5}{|c|}{ Application rate, in acre-feet per acre } & \multirow{2}{*}{$\begin{array}{c}2009-13 \\
\text { average } \\
\text { appli- } \\
\text { cation } \\
\text { rate, in } \\
\text { acre- } \\
\text { feet per } \\
\text { acre }\end{array}$} & \multirow{2}{*}{$\begin{array}{c}2013 \\
\text { average } \\
\text { annual } \\
\text { pre- } \\
\text { cipita- } \\
\text { tion, in } \\
\text { inches }^{3}\end{array}$} & \multirow{2}{*}{$\begin{array}{c}1981- \\
2010 \\
\text { average } \\
\text { annual } \\
\text { pre- } \\
\text { cipita- } \\
\text { tion, in } \\
\text { inches }^{3}\end{array}$} \\
\hline & & & & & 2009 & 2010 & 2011 & 2012 & 2013 & & & \\
\hline Pottawatomie & 188 & 254 & $7,762.71$ & 17,904 & 0.13 & 0.25 & 0.61 & 0.71 & 0.43 & 0.43 & 31.24 & 34.97 \\
\hline Pratt & 697 & 715 & $80,194.42$ & 83,203 & 1.10 & 1.11 & 1.55 & 1.29 & 0.96 & 1.20 & 20.86 & 27.85 \\
\hline Rawlins & 219 & 250 & $20,921.63$ & 18,835 & 0.70 & 0.82 & 0.96 & 1.30 & 1.11 & 0.98 & 18.82 & 21.75 \\
\hline Reno & 787 & 890 & $36,501.69$ & 49,884 & 0.89 & 1.01 & 1.40 & 1.21 & 0.73 & 1.05 & 38.40 & 30.32 \\
\hline Republic & 341 & 432 & $19,288.28$ & 30,308 & 0.58 & 0.59 & 0.52 & 0.90 & 0.64 & 0.65 & 24.98 & 30.89 \\
\hline Rice & 336 & 356 & $12,770.13$ & 17,276 & 0.72 & 0.96 & 1.37 & 1.27 & 0.74 & 1.01 & 40.91 & 28.25 \\
\hline Riley & 67 & 117 & $2,653.54$ & 6,298 & 0.17 & 0.31 & 0.65 & 0.67 & 0.42 & 0.44 & 29.90 & 32.41 \\
\hline Rooks & 39 & 73 & $1,798.65$ & 2,000 & 0.43 & 0.62 & 0.80 & 1.12 & 0.90 & 0.77 & 19.91 & 23.86 \\
\hline Rush & 185 & 282 & $9,634.29$ & 11,464 & 0.83 & 0.82 & 1.20 & 1.14 & 0.84 & 0.97 & 18.59 & 23.63 \\
\hline Russell & 6 & 13 & 4.42 & 15 & 0.36 & 0.41 & 0.59 & 1.13 & 0.29 & 0.56 & 22.23 & 25.96 \\
\hline Saline & 104 & 168 & $1,344.53$ & 3,804 & 0.38 & 0.66 & 0.95 & 1.06 & 0.35 & 0.68 & 22.38 & 31.34 \\
\hline Scott & 467 & 756 & $44,191.84$ & 49,455 & 0.88 & 0.86 & 1.05 & 1.08 & 0.89 & 0.95 & 17.70 & 20.70 \\
\hline Sedgwick & 584 & 680 & $24,197.37$ & 40,852 & 0.68 & 0.85 & 1.21 & 1.07 & 0.59 & 0.88 & 42.61 & 31.20 \\
\hline Seward & 560 & 592 & $189,145.13$ & 128,101 & 1.25 & 1.30 & 1.73 & 1.51 & 1.48 & 1.45 & 9.84 & 19.73 \\
\hline Shawnee & 218 & 256 & $7,715.02$ & 16,846 & 0.12 & 0.33 & 0.57 & 0.72 & 0.46 & 0.44 & 31.90 & 36.20 \\
\hline Sheridan & 683 & 731 & $74,133.80$ & 77,530 & 0.76 & 1.01 & 1.01 & 1.26 & 0.96 & 1.00 & 17.55 & 20.80 \\
\hline Sherman & 824 & 873 & $152,399.21$ & 117,492 & 0.87 & 0.97 & 1.23 & 1.50 & 1.30 & 1.17 & 16.58 & 19.84 \\
\hline Smith & 71 & 168 & $2,002.97$ & 3,462 & 0.50 & 0.43 & 0.45 & 0.85 & 0.58 & 0.56 & 21.40 & 25.97 \\
\hline Stafford & 708 & 740 & $77,462.12$ & 81,885 & 0.94 & 1.17 & 1.56 & 1.31 & 0.95 & 1.19 & 36.22 & 26.03 \\
\hline Stanton & 400 & 715 & $118,503.83$ & 110,284 & 1.12 & 1.17 & 1.36 & 1.29 & 1.07 & 1.20 & 14.49 & 14.60 \\
\hline Stevens & 680 & 758 & $245,210.92$ & 173,490 & 1.30 & 1.26 & 1.72 & 1.55 & 1.41 & 1.45 & 14.62 & 18.43 \\
\hline Sumner & 173 & 192 & $4,267.02$ & 8,809 & 0.43 & 0.70 & 1.12 & 1.10 & 0.48 & 0.77 & 36.52 & 32.46 \\
\hline Thomas & 745 & 787 & $116,499.56$ & 100,884 & 0.68 & 0.89 & 1.12 & 1.35 & 1.15 & 1.04 & 16.87 & 20.43 \\
\hline Trego & 91 & 117 & 4,946.31 & 5,652 & 0.83 & 1.02 & 1.17 & 1.30 & 0.88 & 1.04 & 15.88 & 22.24 \\
\hline Wabaunsee & 95 & 134 & $3,883.56$ & 8,474 & 0.13 & 0.31 & 0.62 & 0.74 & 0.46 & 0.45 & 25.28 & 36.49 \\
\hline Wallace & 330 & 527 & $55,398.72$ & 48,855 & 0.90 & 0.95 & 1.17 & 1.35 & 1.13 & 1.10 & 14.07 & 20.11 \\
\hline Washington & 152 & 238 & $5,030.65$ & 13,016 & 0.42 & 0.31 & 0.28 & 0.71 & 0.39 & 0.42 & 28.29 & 31.62 \\
\hline Wichita & 556 & 835 & $56,551.38$ & 65,874 & 0.82 & 0.85 & 0.90 & 0.98 & 0.86 & 0.88 & 10.77 & 18.44 \\
\hline Wilson & 15 & 52 & 488.24 & 1,398 & 0.26 & 0.23 & 0.74 & 0.49 & 0.35 & 0.41 & 40.51 & 40.69 \\
\hline Woodson & 1 & 1 & 38.08 & 114 & 0.00 & 0.42 & 0.82 & 0.64 & 0.33 & 0.44 & 40.16 & 42.12 \\
\hline Wyandotte & 13 & 17 & 341.17 & 473 & 0.48 & 0.46 & 0.80 & 1.09 & 0.72 & 0.71 & 34.01 & 38.70 \\
\hline State Total & 24,105 & 30,271 & $3,304,425$ & $3,044,601$ & 0.99 & 1.08 & 1.36 & 1.32 & 1.09 & 1.19 & - & - \\
\hline
\end{tabular}

\footnotetext{
${ }^{1}$ Water use does not include surface water withdrawn under ditch irrigation water rights and by irrigation districts (table 12).

${ }^{2}$ Acreage does not include land irrigated under ditch irrigation water rights and irrigation districts (table 12).

${ }^{3}$ The 2013 annual total precipitation and the current 30-year climatic normal (based on the period 1981-2010) for the 105 Kansas counties are from the "Kansas statistical abstract 2013" (Institute for Policy and Social Research, 2014).
} 
Table 4. Comparison of water use and acres irrigated by meter status and Kansas irrigation water-use analysis regions, 2013.

[GMD, groundwater management district]

\begin{tabular}{|c|c|c|c|c|c|c|c|c|c|c|}
\hline \multirow[b]{2}{*}{$\begin{array}{l}\text { Kansas irrigation } \\
\text { water-use analysis } \\
\text { regions (fig. 1) }\end{array}$} & \multicolumn{4}{|c|}{ Metered } & \multicolumn{4}{|c|}{ Nonmetered } & \multirow[b]{2}{*}{$\begin{array}{c}\text { Total } \\
\text { number } \\
\text { of active } \\
\text { points of } \\
\text { diversion } \\
\text { reporting } \\
\text { water use } \\
\text { for the } \\
\text { year } 2013\end{array}$} & \multirow[b]{2}{*}{$\begin{array}{l}\text { Percent } \\
\text { metered }\end{array}$} \\
\hline & $\begin{array}{c}\text { Total } \\
\text { number } \\
\text { of active } \\
\text { points of } \\
\text { diversion } \\
\text { reporting } \\
\text { water use } \\
\text { for the } \\
\text { year } 2013\end{array}$ & $\begin{array}{l}2013 \text { water } \\
\text { use, in } \\
\text { acre feet }\end{array}$ & $\begin{array}{c}2013 \\
\text { reported } \\
\text { irrigated } \\
\text { acres }^{2}\end{array}$ & $\begin{array}{c}2013 \\
\text { appli- } \\
\text { cation } \\
\text { rate, in } \\
\text { acre- } \\
\text { feet per } \\
\text { acre }\end{array}$ & $\begin{array}{c}\text { Total } \\
\text { number } \\
\text { of active } \\
\text { points of } \\
\text { diversion } \\
\text { reporting } \\
\text { water use } \\
\text { for the } \\
\text { year } 2013\end{array}$ & $\begin{array}{c}2013 \\
\text { water } \\
\text { use, in } \\
\text { acre } \\
\text { feet }^{1}\end{array}$ & $\begin{array}{c}2013 \\
\text { reported } \\
\text { irrigated } \\
\text { acres }^{2}\end{array}$ & $\begin{array}{c}2013 \\
\text { appli- } \\
\text { cation } \\
\text { rate, in } \\
\text { acre- } \\
\text { feet per } \\
\text { acre }\end{array}$ & & \\
\hline $\begin{array}{l}\text { Western Kansas } \\
\text { GMD No. } 1\end{array}$ & 1,387 & 161,971 & 173,562 & 0.93 & 244 & 23,923 & 25,830 & 0.93 & 1,631 & 85.0 \\
\hline $\begin{array}{l}\text { Southwest Kansas } \\
\text { GMD No. } 3\end{array}$ & 7,822 & $1,868,181$ & $1,460,525$ & 1.28 & 15 & 2,642 & 2,565 & 1.03 & 7,837 & 99.8 \\
\hline $\begin{array}{l}\text { Equus Beds GMD } \\
\text { No. } 2\end{array}$ & 1,483 & 71,334 & 113,352 & 0.63 & 114 & 8,247 & 14,923 & 0.55 & 1,597 & 92.9 \\
\hline $\begin{array}{l}\text { Big Bend GMD } \\
\text { No. } 5\end{array}$ & 4,036 & 423,933 & 440,410 & 0.96 & 10 & 836 & 1,249 & 0.67 & 4,046 & 99.8 \\
\hline $\begin{array}{l}\text { Remainder of } \\
\text { Central Kansas }\end{array}$ & 3,204 & 134,016 & 221,814 & 0.60 & 112 & 5,461 & 10,647 & 0.51 & 3,316 & 96.6 \\
\hline All Eastern Kansas & 866 & 32,042 & 74,278 & 0.43 & 152 & 6,099 & 11,313 & 0.54 & 1,018 & 85.1 \\
\hline State Total & 23,421 & $3,253,184$ & $2,975,001$ & 1.09 & 684 & 51,241 & 69,600 & 0.74 & 24,105 & 97.2 \\
\hline
\end{tabular}

Table 5. Comparison of water use and acres irrigated by meter status and regional planning area, 2013.

$[-$, none reported $]$

\begin{tabular}{|c|c|c|c|c|c|c|c|c|c|c|}
\hline \multirow[b]{2}{*}{$\begin{array}{l}\text { Regional planning } \\
\text { area (fig. 2) }\end{array}$} & \multicolumn{4}{|c|}{ Metered } & \multicolumn{4}{|c|}{ Nonmetered } & \multirow[b]{2}{*}{$\begin{array}{c}\text { Total } \\
\text { number } \\
\text { of active } \\
\text { points of } \\
\text { diversion } \\
\text { reporting } \\
\text { water use } \\
\text { for the } \\
\text { year } 2013\end{array}$} & \multirow[b]{2}{*}{$\begin{array}{l}\text { Per- } \\
\text { cent } \\
\text { me- } \\
\text { tered }\end{array}$} \\
\hline & $\begin{array}{c}\text { Total } \\
\text { number } \\
\text { of active } \\
\text { points of } \\
\text { diversion } \\
\text { reporting } \\
\text { water use } \\
\text { for the } \\
\text { year } 2013\end{array}$ & $\begin{array}{c}2013 \text { water } \\
\text { use, in acre } \\
\text { feet }^{1}\end{array}$ & $\begin{array}{c}2013 \\
\text { reported } \\
\text { irrigated } \\
\text { acres }^{2}\end{array}$ & $\begin{array}{c}2013 \\
\text { appli- } \\
\text { cation } \\
\text { rate, in } \\
\text { acre- } \\
\text { feet } \\
\text { per } \\
\text { acre }\end{array}$ & $\begin{array}{c}\text { Total } \\
\text { number } \\
\text { of active } \\
\text { points of } \\
\text { diversion } \\
\text { reporting } \\
\text { water use } \\
\text { for the } \\
\text { year } 2013\end{array}$ & $\begin{array}{l}2013 \text { water } \\
\text { use, in } \\
\text { acre feet }^{1}\end{array}$ & $\begin{array}{c}2013 \\
\text { reported } \\
\text { irrigated } \\
\text { acres }^{2}\end{array}$ & $\begin{array}{c}2013 \\
\text { appli- } \\
\text { cation } \\
\text { rate, in } \\
\text { acre- } \\
\text { feet per } \\
\text { acre }\end{array}$ & & \\
\hline Cimarron & 2,876 & $883,519.99$ & 667,024 & 1.32 & 3 & 782.62 & 1,020 & 0.77 & 2,879 & 99.9 \\
\hline Equus-Walnut & 1,958 & $80,551.46$ & 131,455 & 0.61 & 190 & $11,913.30$ & 21,767 & 0.55 & 2,148 & 91.2 \\
\hline Great Bend Prairie & 4,626 & $458,040.69$ & 479,965 & 0.95 & 14 & $1,103.35$ & 1,512 & 0.73 & 4,640 & 99.7 \\
\hline Kansas & 1,324 & $58,989.46$ & 121,298 & 0.49 & 154 & $5,891.00$ & 11,953 & 0.49 & 1,478 & 89.6 \\
\hline Red Hills & 428 & $31,980.04$ & 38,826 & 0.82 & 1 & 46.66 & 135 & 0.35 & 429 & 99.8 \\
\hline
\end{tabular}


Table 5. Comparison of water use and acres irrigated by meter status and regional planning area, 2013.-Continued

$[-$, none reported $]$

\begin{tabular}{|c|c|c|c|c|c|c|c|c|c|c|}
\hline \multirow[b]{2}{*}{$\begin{array}{l}\text { Regional planning } \\
\text { area (fig. 2) }\end{array}$} & \multicolumn{4}{|c|}{ Metered } & \multicolumn{4}{|c|}{ Nonmetered } & \multirow[b]{2}{*}{$\begin{array}{c}\text { Total } \\
\text { number } \\
\text { of active } \\
\text { points of } \\
\text { diversion } \\
\text { reporting } \\
\text { water use } \\
\text { for the } \\
\text { year } 2013\end{array}$} & \multirow[b]{2}{*}{$\begin{array}{c}\text { Per- } \\
\text { cent } \\
\text { me- } \\
\text { tered }\end{array}$} \\
\hline & $\begin{array}{c}\text { Total } \\
\text { number } \\
\text { of active } \\
\text { points of } \\
\text { diversion } \\
\text { reporting } \\
\text { water use } \\
\text { for the } \\
\text { year } 2013\end{array}$ & $\begin{array}{c}2013 \text { water } \\
\text { use, in acre } \\
\text { feet }^{1}\end{array}$ & $\begin{array}{c}2013 \\
\text { reported } \\
\text { irrigated } \\
\text { acres }^{2}\end{array}$ & $\begin{array}{c}2013 \\
\text { appli- } \\
\text { cation } \\
\text { rate, in } \\
\text { acre- } \\
\text { feet } \\
\text { per } \\
\text { acre }\end{array}$ & $\begin{array}{c}\text { Total } \\
\text { number } \\
\text { of active } \\
\text { points of } \\
\text { diversion } \\
\text { reporting } \\
\text { water use } \\
\text { for the } \\
\text { year } 2013\end{array}$ & $\begin{array}{l}2013 \text { water } \\
\text { use, in } \\
\text { acre feet }^{1}\end{array}$ & $\begin{array}{c}2013 \\
\text { reported } \\
\text { irrigated } \\
\text { acres }^{2}\end{array}$ & $\begin{array}{c}2013 \\
\text { appli- } \\
\text { cation } \\
\text { rate, in } \\
\text { acre- } \\
\text { feet per } \\
\text { acre }\end{array}$ & & \\
\hline Smoky Hill-Saline & 478 & $13,266.59$ & 25,081 & 0.53 & 12 & 653.86 & 1,048 & 0.62 & 490 & 97.6 \\
\hline $\begin{array}{l}\text { Solomon-Repub- } \\
\text { lican }\end{array}$ & 1,218 & $50,576.90$ & 74,007 & 0.68 & 3 & 50.58 & 144 & 0.35 & 1,221 & 99.8 \\
\hline Upper Arkansas & 5,152 & $1,010,424.58$ & 816,859 & 1.24 & 22 & $3,927.05$ & 2,735 & 1.44 & 5,174 & 99.6 \\
\hline State Total & 23,421 & $3,253,183.86$ & $2,975,001$ & 1.09 & 684 & $51,240.89$ & 69,600 & 0.74 & 24,105 & 97.2 \\
\hline
\end{tabular}

${ }^{1}$ Water use does not include surface water withdrawn under ditch irrigation water rights and by irrigation districts (table 12).

${ }^{2}$ Acreage does not include land irrigated under ditch irrigation water rights and irrigation districts (table 12).

heading, "Combination alfalfa, corn, grain sorghum, soybeans, or wheat." When an irrigator specifies the code for "more than one type of crop" or "double crop" then the water used, irrigated acres, and application rates for 2013 are combined under the column heading "More than one type of crop specified" in table 6. The "Other" column in table 6 is a combination of the following crop types: oats, barley, rye, dry beans, sunflowers, golf course and (or) sports fields, truck farm, orchard, nursery, cotton, grapes, or other. Any water-use report where the crop code was left blank is listed under the column heading "not specified on 2013 water-use report." The same statistics, aggregated by regional planning area (fig. 2) and county, respectively, are shown in tables 7 and 8 .

Water use by irrigation method for 2013 is summarized by water-use analysis region (fig. 1) in table 9. The instructions on the 2013 irrigation water-use report (see appendix for blank report) request that a code be entered for the type of irrigation system used by each point of diversion in 2013 . The irrigation system codes are integer values for the following system types: center pivot with drop nozzles; center pivot sprinkler; center pivot and flood; flood; drip (subsurface irrigation); drip and other system; sprinkler other than center pivot; and other. The "Subsurface drip, and combination of drip and other system" column in table 9 is a combination of (1) the drip (subsurface irrigation) system type and (2) drip and other system types. The 2013 water use by irrigation method by regional planning area (fig. 2) is provided in table 10 . The 2013 water use by irrigation method by county is provided in table 11 . Water-use efficiency can differ dramatically by irrigation method.

\section{Surface-Water Ditch Companies and Irrigation Districts}

A number of private ditch companies with irrigation water rights are in the southwest part of Kansas in the counties of Hamilton, Finney, and Kearny. Irrigation districts with water rights are in the north-central part of Kansas in Cloud, Jewell, Mitchell, Norton, Osborne, Ottawa, Phillips, Republic, Rooks, and Smith Counties. Reports filed by these systems contain total water use and total acres irrigated and are tabled separately from the other water rights because the amount of water applied to irrigated acres cannot be accurately associated with the point of diversion (table 12). Frequently, these acres are irrigated by both ditch water and individual water rights; therefore, the number of acres listed in table 12 should not be combined with the State totals.

\section{Summary}

This report documents 2013 irrigation water use in Kansas. The tables provided in this report were prepared using self-reported data from irrigation reports filed for the year 2013 with the Kansas Department of Agriculture, Division of Water Resources. The published regional and county-level statistics from the previous 4 years (2009-12) are shown with the 2013 statistics and are used to calculate a 5-year average. An overall Kansas average and regional averages also are calculated and presented. Total reported irrigation water use in 2013 was 3.3 million acre-feet of water applied to 3.0 million irrigated acres. 


\section{References Cited}

Di Luzio, Mauro, Johnson, G.L., Daly, Christopher, Eischeid, J.K., and Arnold, J.G., 2008, Constructing retrospective gridded daily precipitation and temperature datasets for the conterminous United States: Journal of Applied Meteorology and Climatology, v. 47, no. 2, p. 475-497.

High Plains Regional Climate Center [2015], Climate data: High Plains Regional Climate Center Web page, accessed September 11, 2015, at http://www.hprcc.unl.edu/maps. php? map $=$ Normals.

Institute for Policy and Social Research, 2014, Kansas statistical abstract 2013 (48th ed.): Institute for Policy and Social Research, 583 p., accessed September 25, 2015, at http:// ipsr.ku.edu/ksdata/ksah/KSA48.pdf.

Kansas Department of Agriculture, 2015, Kansas farm facts: Kansas Department of Agriculture, 60 p., accessed September 29, 2015, at http://agriculture.ks.gov/ docs/default-source/ag-marketing/kansas-farm-factsfebruary-13-2015.pdf.

Kansas Department of Agriculture, Division of Water Resources, 1989-2012, Kansas irrigation water use: Topeka, Kans, [published annually since 1989, pagination and authorship varies, available on request from the Kansas Department of Agriculture].

Kansas Department of Agriculture, Division of Water Resources, 2015, Water use data collection and use fact sheet: Topeka, Kans., 2 p., accessed September 29, 2015 , at http://agriculture.ks.gov/docs/default-source/dwrwater-appropriation-documents/waterusedatacollection2015b0d5d4002e6262e1aa5bff0000620720.pdf.
Kenny, J.F., 2014, Public-supply water use in Kansas, 1990-2012: U.S. Geological Survey Fact Sheet 2014-3116, 4 p., accessed September 25, 2015, at http://dx.doi.org/ $10.3133 /$ fs 20143116 .

Kenny, J.F., and Juracek, K.E., 2013, Irrigation trends in Kansas, 1991-2011: U.S. Geological Survey Fact Sheet 2013-3094, 4 p., accessed accessed September 25, 2015, at http://pubs.usgs.gov/fs/2013/3094/.

National Agricultural Statistics Service, 2014, 2012 Census of agriculture, United States summary and State data: U.S. Department of Agriculture, v. 1, part 51, 695 p., accessed September 25, 2015, at http://www.agcensus.usda.gov/ Publications/2012/.

Perry, C.A., 2006, Effects of irrigation practices on water use in the Groundwater Management Districts within the Kansas High Plains, 1991-2003: U.S. Geological Survey Scientific Investigations Report 2006-5069, 93 p., accessed September 25, 2015, at http://pubs.usgs.gov/sir/2006/5069/.

Peters, A.J., and Rasmussen, T.J., 2014, Summary of hydrologic conditions in Kansas, 2013 water year: U.S. Geological Survey Fact Sheet 2014-3061, 6 p., accessed September 25, 2015, at http://dx.doi.org/10.3133/fs20143061.

PRISM Climate Group, 2015, PRISM climate data: Oregon State University Northwest Alliance for Computational Science and Engineering database, accessed September 28, 2015, at http://prism.oregonstate.edu/.

U.S. Drought Monitor, 2014, Tabular data archiveUnited States drought monitor: accessed September 29, 2015, at http://droughtmonitor.unl.edu/MapsAndData/ DataTables.aspx. 


\section{Tables 6-12}

An Excel file containing the tables is available at http://dx.doi.org/10.3133/ds981.

Table 6. Water use, acres irrigated, and average application rate by crop and Kansas irrigation water-use analysis region, 2013.

Table 7. Water use, acres irrigated, and average application rate by crop and regional planning area, 2013.

Table 8. Water use, acres irrigated, and average application rate by crop and county, 2013.

Table 9. Water use, acres irrigated, and average application rate by type of irrigation system by Kansas irrigation water-use analysis regions, 2013.

Table 10. Water use, acres irrigated, and average application rate by type of irrigation system by regional planning area, 2013.

Table 11. Water use, acres irrigated, and average application rate by type of irrigation system by county, 2013.

Table 12. Water use and acres irrigated by ditch companies and irrigation districts in Kansas, 2013.

\section{Appendix}

The Kansas Department of Agriculture's Division of Water Resources water-use report can be downloaded at http://dx.doi.org/10.3133/ds981.

Manuscript approved February 26, 2016

For more information about this publication, contact:

Director

USGS Kansas Water Science Center

4821 Quail Crest Place

Lawrence, KS 66049

(785) 842-9909

Or visit the Kansas Water Science Center Web site:

http://ks.water.usgs.gov 



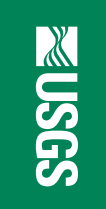

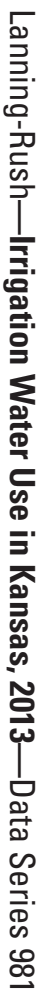

ISSN 2327-638X (online) 